http://jmscr.igmpublication.org/home/

ISSN (e)-2347-176x ISSN (p) 2455-0450

crossref DOI: https://dx.doi.org/10.18535/jmscr/v10i1.25

Journal Of Medical Science And Clinical Research

\title{
A Case Report On MR Imaging of Multicystic Encephalomalacia of Infancy
}

\author{
Authors \\ Dr Ila Desai ${ }^{1}$, Dr Arjav Patel ${ }^{2}$, Dr Riddhi Gohil ${ }^{3}$, Dr Reecha Prasad ${ }^{4}$ \\ ${ }^{1}$ Professor Department of Radiodiagnosis, B.J. Medical College Ahmedabad \\ ${ }^{2}$ 2nd Year Radiology Resident, B.J. Medical College Ahmedabad \\ ${ }^{3}$ 2nd Year Radiology Resident, B.J. Medical College Ahmedabad \\ ${ }^{4}$ 2nd Year Radiology Resident, GCRI, B.J. Medical College Ahmedabad
}

\begin{abstract}
Multicystic encephalomalacia is a diffuse lesion of the brain in which cerebral parenchyma is replaced by cysts of varying sizes in the perinatal period. It is stressed that multiple cystic encephalomalacia has a characteristic appearance on MRI and be used to detect it at an early stage. This is a case report of an infant showing multiple cysts spaces in brain secondary to hypoxic insult.

Keywords: Hypoxia, Multicystic Encephalomalacia, MRI.
\end{abstract}

\section{Introduction}

Perinatal hypoxic ischemic brain injury is the leading cause of neonatal encephalopathy and accounts for between $6 \%$ and $8 \%$ of cerebral palsy worldwide. Magnetic resonance imaging is the key imaging modality in the evaluation of suspected hypoxic ischemic brain injury.

Multicystic encephalomalacia is a condition defined anatomically by the presence of multiple cystic cavities of variable size in the greater part of both cerebral hemispheres. It is associated with profound neuromotor delay and consequent grave prognosis. Typically, the posterior fossa is spared with usually no connection to the ventricular system. It may be clinically silent; and is discovered when the infant fails to achieve the early milestones of neuromotor development. Various other causes which include meningitis and twin-to-twin transfusion can lead to this disorder.

\section{Case Report}

A 6 month-old baby boy with a history of placental insufficiency and premature birth at 7 months of gestational age was referred to department of radio diagnosis with complain of failure in motor development.

MRI study of the brain was performed on a $1.5 \mathrm{~T}$ MR scanner, with T1, T2 weighted, FLAIR sequences on three planes. 


\section{JMSCR Vol||10||Issue ||01||Page 144-147||January}

\section{Imaging Findings}

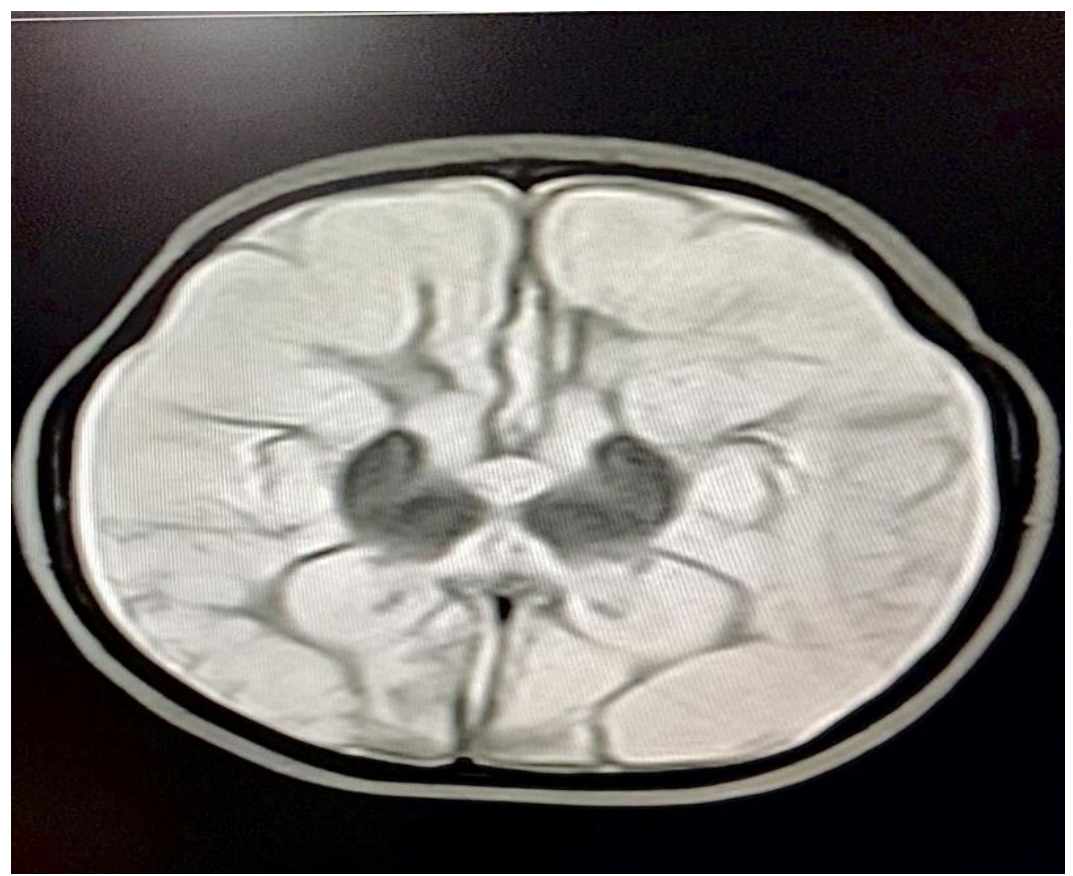

Axial T2-weighted MR image shows multiple cysts of various sizes, with a signal intensity almost isointense to CSF, which are separated by linear septae composed of glial cells and some viable neurons. The immediate periventricular white matter is spared. The overlying cerebral cortex is thinned. There is associated ex vacuo dilatation of third and lateral ventricles are associated, and diencephalic structures are preserved.

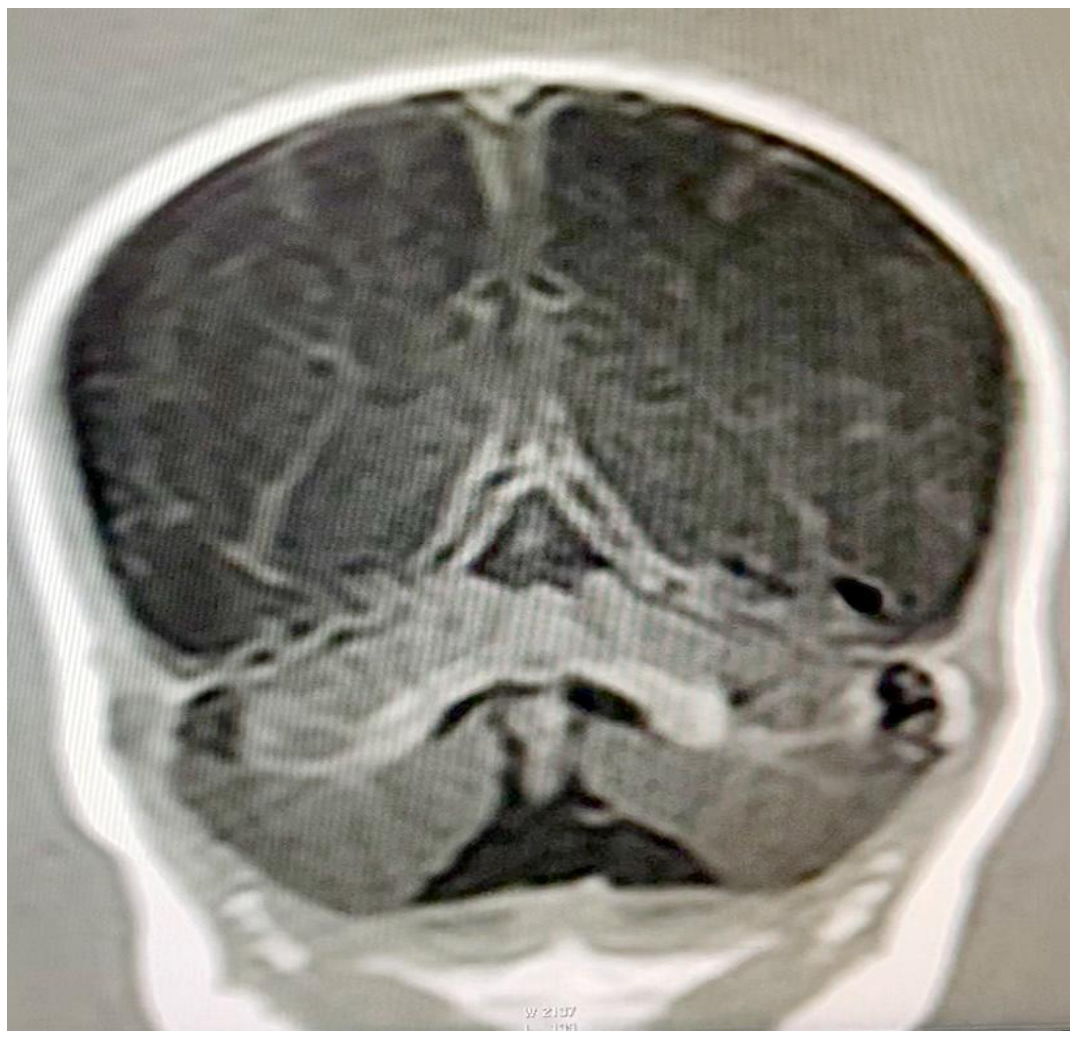

Coronal FLAIR MR image shows multiple cysts of various sizes, with a signal intensity isointense to CSF, which are separated by linear septae composed of glial cells and some viable neurons. The immediate periventricular white matter and diencephalic structures are spared. The overlying cerebral cortex is thinned. Note the ex vacuo dilatation of the ventricles. There is relative sparing of cerebellum is noted. 


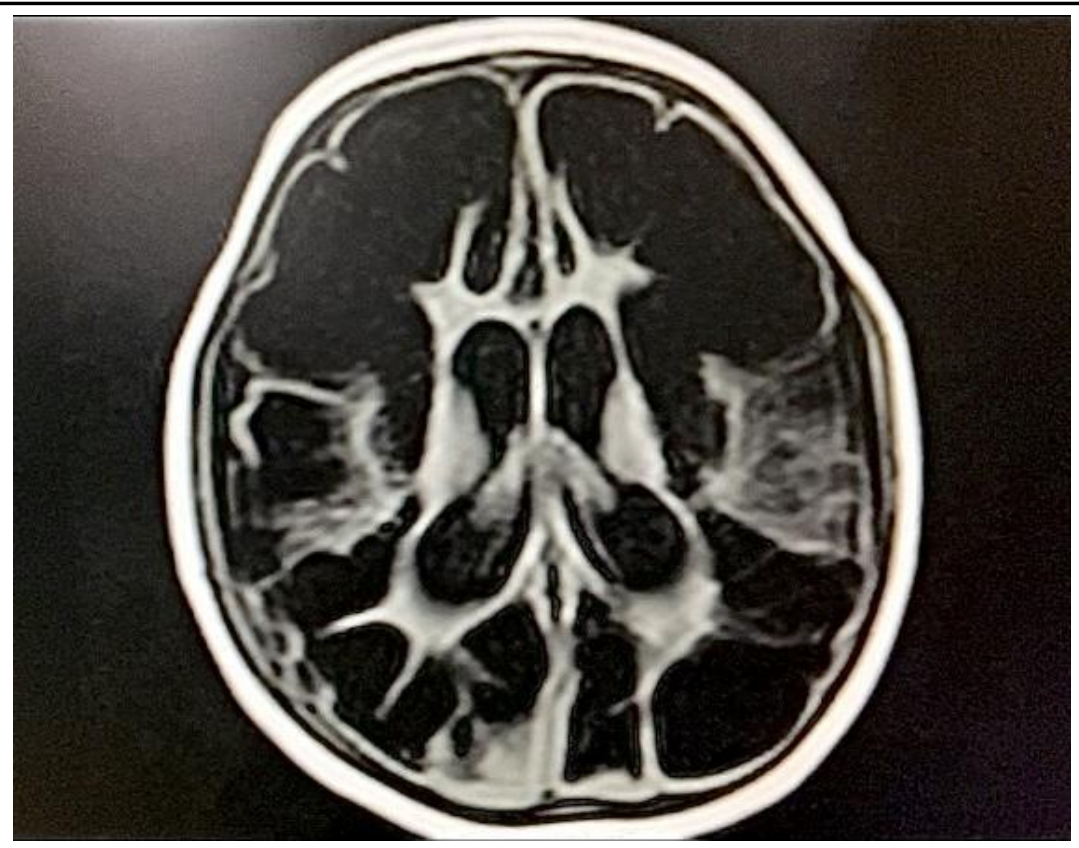

Axial FLAIR MR image shows multiple cysts of various sizes, with a signal intensity almost isointense to $\mathrm{CSF}$, which are separated by linear septae with relative sparring of inferior temporal lobes.

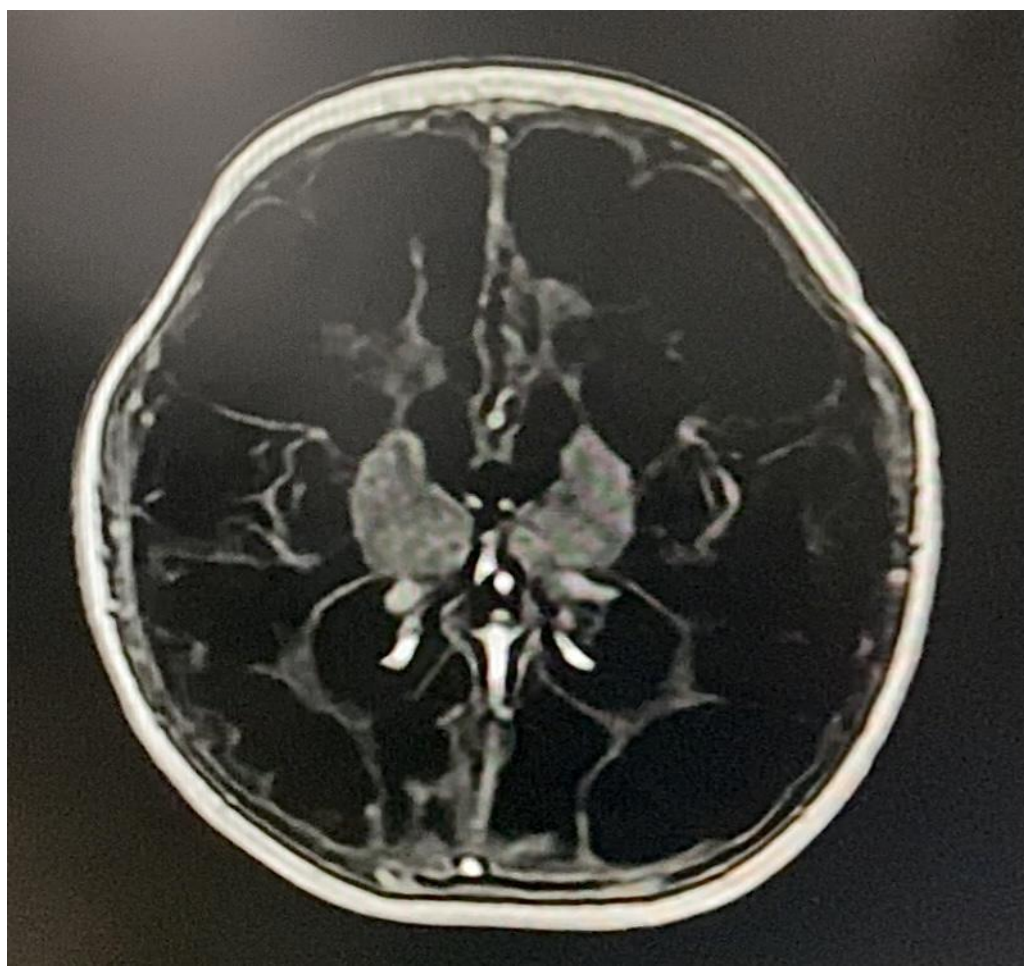

Axial T1-weighted contrast MR image shows multiple cysts of various sizes, with a signal intensity almost isointense to CSF, which are separated by linear septae with relative sparing of basal nuclei on both sides.

\section{Discussion}

Multicystic encephalomalacia results from a diffuse insult to the brain late in gestation, during birth, or after birth. Multicystic encephalomalacia may occur as a result of ischemia, infection, or developmental insults.
In very severe cases of hypoxia or total anoxia, hypoxia induced brain injury can extend beyond the watershed or boundary zones to involve major vascular territories completely. Multiple cystic cavities of variable size form in the necrotic area, separated from one another by glial septations. 
The cerebrum becomes infarcted more globally and diffusion signal abnormality may be difficult to appreciate as the entire cerebrum can show restricted diffusion with relatively no normal cerebral lobe to compare with. As this injury evolves, there is progressive loss of cerebral volume with extensive irreversible neuronal destruction, vacuolation, cystic change or spongiosis which results in Multilobar cystic encephalomalacia.

The location of the lesions varies with the nature of the insult. If caused by thromboembolic infarction, the affected area will be in the distribution of a major cerebral artery. If the injury is a result of partial asphyxia, it will tend to be located in the cortex and peripheral white matter, primarily in the watershed areas. When the insult is very severe, only the immediate periventricular white matter may be spared.

There is usually marked ventriculomegaly because of ex vacuo dilatation from the extensive surrounding white matter destruction. Relative sparing of the immediate periventricular white matter and the basal nuclei may be seen. This is classified as the first subtype of cystic encephalomalacia. In cases where the insult was an acute, severe and sustained hypoxic event, the basal nuclei are more likely to be involved, presenting with the second subtype of cystic encephalomalacia

Differential diagnosis of Multilobar cystic encephalomalacia includes encephaloclastic porencephaly, hydranencephaly. The differentiation of an encephaloclastic porencephaly is not difficult by its smooth-walled, fluid-filled cavity (a porencephalic cyst) in contrary to the encephalomalacic cavity which contains septations and an irregular wall composed primarily of reactive astrocytes. Hydranencephaly is characterized by the destruction and resorption of most of the brain mantle except frontal regions, and by CSF-filled thin-walled cerebral hemispheres.

\section{Conclusion}

MRI has been utilised in the evaluation of hypoxia brain injury in children with cerebral palsy. MRI is a clinically independent valuable biomarker of hypoxi brain injury in the setting of neonatal encephalopathy. MRI retrospectively gives correlation between the pattern of cerebral injury with possible clinicopathogenesis.

\section{References}

1. Keenan HT, Runyan DK, Marshall SW, et al. A population-based study of inflicted traumatic brain injury in young children. JAMA. 2003;290(5):621-626. doi: 10.1001/jama.290.5.621.

2. Imaging of Multicystic Encephalomalacia of Infancy (MICE) : A Case Report

3. December 2014 International Journal of Biomedical and Advance Research 5(11):566DOI:10.7439/ijbar.v5i11.344

4. Theodore AD, Chang JJ, Runyan DK, et al. Epidemiologic features of the physical and sexual maltreatment of children in the Carolinas. Pediatrics. 2005;115(3):e331e337. doi: 10.1542/peds.2004-1033.

5. Barkovich AJ. Pediatric Neuroimaging. Lippincott-Raven Publishers, Philadelphia (2000)

6. Sie LT, van der Knaap MS, Oosting J, et al. MR patterns of hypoxic-ischemic brain damage after prenatal, perinatal or postnatal asphyxia. Neuropediatrics. 2000;31(3):128-136. doi: 10.1055/s-20007496. 